\title{
CHALLENGE OF BENCHMARKING SIMULATION CODES FOR THE LANL BEAM-HALO EXPERIMENT *
}

\author{
W.P.Lysenko $^{1}$, J.Qiang ${ }^{2}$, R.W.Garnett ${ }^{1}$, and T. P. Wangler ${ }^{1}$ \\ ${ }^{1}$ Los Alamos National Laboratory \\ ${ }^{2}$ Lawrence Berkeley National Laboratory
}

\begin{abstract}
We compare macroparticle simulations with beam-profile measurements from a proton beam-halo experiment in a study of beam-halo formation in mismatched beams in a 52-quadrupole periodic-focusing channel. The lack of detailed measurement of the initial distribution is an important issue for being able to make reliable predictions of the halo. We have found earlier that different initial distributions with the same CourantSnyder parameters and emittances produce similar matched-beam profiles, but different mismatched-beam profiles in the transport system. Also, input distributions with greater population in the tails produce larger rates of emittance growth. We have concluded that using only the known Courant-Snyder parameters and emittances as input parameters is insufficient information for reliable simulations of beam halo formed in mismatched beams. The question is how to obtain the best estimate of the input beam distribution needed for more accurate simulations. In this paper, we investigate a new least squares fitting procedure, which is applied to the simulations and used to determine the injected beam distribution in an attempt to obtain a more accurate description of halo formation than from simulation alone.
\end{abstract}

\section{INTRODUCTION}

This paper is concerned with the comparison of selfconsistent macroparticle simulations, including spacecharge forces using the macroparticle simulation code IMPACT[1], with experimental measurements of the beam profiles in a high-current proton beam. The measurements were made in a beam-transport channel using a $6.7-\mathrm{MeV}$ proton beam at the Low Energy Demonstration Accelerator (LEDA) facility [2] at Los Alamos National Laboratory. A major goal of the experiment was to validate the beam-dynamics simulations of beam halo. Of particular importance was the validation of the space-charge routine in IMPACT. We have observed good agreement comparing direct simulations and profile measurements for the matched beam but have been less successful in obtaining good agreement for the mismatched beams [3]. We have concluded that the beam profiles for mismatched beams are very sensitive to the initial distribution. We now investigate the use of a least squares fitting procedure applied to the RFQ simulation results to obtain an improved estimate of the initial beam distribution (at the exit of the RFQ). We suggest that this fitting procedure may provide an improved estimate of the real beam distribution at the output of the RFQ, because it allows us to correct for unknown errors upstream of the beamtransport line that may prevent us from deducing accurately the RFQ output distribution using simulations alone. Improving our prediction of the RFQ output distribution would provide a more accurate predictive capability for the beam halo evolution in a high energy proton linac.

\section{BEAM HALO EXPERIMENT}

The LEDA facility consists of a 75-keV DC injector, a low-energy beam transport (LEBT) system, and a 350$\mathrm{MHz}$ radiofrequency quadrupole (RFQ), which accelerates the proton beam to $6.7 \mathrm{MeV}$. A schematic diagram of the LEDA beam-halo experiment transport system that follows the RFQ is shown in Fig. 1[4]. The transport system consists of 52 magnetic quadrupoles with alternating polarity (FODO Lattice) to provide strong periodic transverse focusing. Transverse beam profiles were measured using beam-profile detectors[5] located in the middle of the drift spaces after quadrupoles 4, 20, 22, 24, 26, 45, 47, 49 and 51. The first four quadrupole gradients were independently adjusted to match the beam, by producing equal rms sizes at the beam-profile detectors. The gradients were also adjusted to produce approximately pure mismatches in either a breathing or a quadrupole mode. The beam current was varied over a range from 16 to $100 \mathrm{~mA}$. In this paper we report results at $75-\mathrm{mA}$.

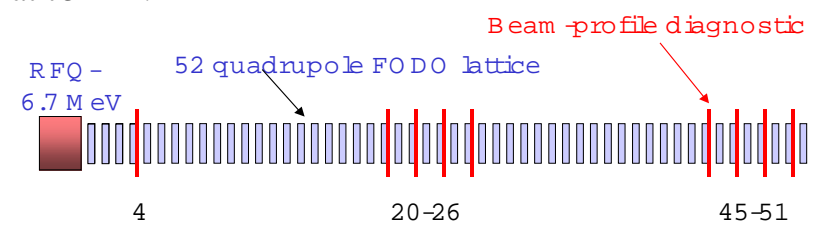

FIG. 1. Block diagram of the 52-quadrupole-magnet lattice showing the nine locations of beam-profile scanners. The first four quadrupoles upstream of scanner 4 are adjusted to match or mismatch the beam.

\section{OLD SIMULATION RESULTS}

We obtained rms beam widths at each of the scanners and rms emittances at scanners 20 and 45 . The emittances 
as well as the Courant-Snyder parameters were deduced at scanners 20 and 45 using the rms values from the four associated profile monitors $(20,22,24$, and 26 , for the value at 20 , and $45,47,49$, and 51 for the value at 45). The measured results for the mismatched beams were compared with the maximum amplitude predictions of a particle-core model [6], and maximum emittance growth predictions of a free energy model [7], assuming complete transfer of the free energy. The experimental results strongly supported both analytic models[8]. We have observed good agreement between simulations and profile measurements for the matched beam, but have been less successful in obtaining good agreement for the mismatched beams. We believe that the main cause of the poor agreement for the mismatched cases lies in a lack of detailed knowledge of the initial distribution in phase space. Given that we were unable to measure the input distribution, our approach to the simulations was to generate four different initial distributions at the entrance of the beam-transport channel, all with the same CourantSnyder ellipse parameters and emittances; these parameters were deduced from the measurements. The assumed input distributions were: 1) 6D Waterbag, 2) 6D Gaussian, 3) Double Gaussian, and 4) a distribution called LEBT/RFQ, generated from a simulation through the LEBT and RFQ, starting at the plasma surface at the exit of the ion source. All four distributions were scaled to produce the correct initial Courant-Snyder parameters and emittances. These four distributions differ qualitatively with respect to their initial halo, i.e. initial population of the outer tails of the beam. Best agreement for the observed emittance growth was obtained with the Double Gaussian, which had the largest population in the tails. This is a result that would be expected from the particlecore model [6], since most of the halo particles have initial amplitudes that lie outside the core. However, none of these initial distributions yield good agreement with measured beam profiles for the mismatched case. We have concluded that knowledge of the initial particle distribution, especially the density in the tails, is important for accurate simulations of the beam halo.

The question that remains is how to obtain reliable simulations for mismatched beams when the input distribution is not measured. A possible solution is suggested by recent results obtained from the analysis of earlier quadrupole scan measurements used to characterize the beam at the exit of the RFQ [9]. In that case the authors concluded that unknown beamlineparameter errors could lead to differences between the real and the simulation results at the output of the RFQ. The simplest assumption is that the main errors involve only the second moments, i.e. the Courant-Snyder parameters and the rms emittances. Therefore the authors modified the simulation results by adjusting the simulated RFQ output particle coordinates to change the second moments of the simulated RFQ output beam to give a best least-squares fit to all the measured profiles. This procedure produced excellent agreement between measured and simulated profiles. We would like to investigate whether a similar method would also produce improved agreement for the beam halo experiment.

\section{NEW RESULTS WITH LEAST-SQUARES FITTING}

Our hypothesis is to assume that our simulation codes do contain the relevant physics. Discrepancies are most likely caused by the fact that we do not know the precise parameters for the LEBT and for the as-built RFQ, particularly the axial voltage distribution. Therefore, the real beam distribution that is injected into the beam transport channel after the RFQ is not precisely known. For this reason we have investigated whether modifications to the beam generated by RFQ simulations would improve the agreement between the simulated and measured beam profiles. We have used a modified version of the fitting code described in ref. [9] in which an IMPACT simulation of the halo channel is controlled by a nonlinear optimizer. The input beam is described by the six transverse parameters (Courant-Snyder parameters $\alpha$, $\beta$, and emittances $\varepsilon$ in both $\mathrm{x}$ and $\mathrm{y}$ ). The optimizer varies the six parameters and attempts to minimize the differences between the simulated profiles at the wirescanner positions and the measured profiles.

The input beams for these simulations are generated by starting with the particle distribution from our LEBT/RFQ simulation. This distribution is distorted by applying a certain linear transformation [9] to the particle phase-space coordinates that results in the beam having the six Courant-Snyder parameters requested by the nonlinear optimizer; this procedure should retain much of the higher-order structure present in the original RFQ simulation.

We fitted only to the five wire scanner profiles between 4 to 26 (10 profiles) for the matched case. Using the modified initial distribution, the simulated and measured profiles agree well for all the scanners for the matched beam. Figure 2 shows results for the matched case and the mismatched case for mismatch parameter $\mu=1.5$, where $\mu$ is defined in Ref. [8]. Rms deviations for the matched case (difference between experimental and simulated profiles) are small, about $2 \%$. However, using this beam to simulate the mismatched cases has not yet produced as good agreement between the simulated and measured profiles.

\section{CONCLUSIONS}

We report on an investigation of a new least-squares fitting procedure, which is applied to the simulations in an attempt to obtain a more accurate description of the initial particle distribution. The fits were applied to a subset of the matched beam profiles. We find that the resulting profiles for the matched beam at all the scanners agree well with the measured ones, but more work is required to improve the agreement for the mismatched beams. We plan to investigate this situation further by fitting the mismatched data or fitting the matched and mismatched 

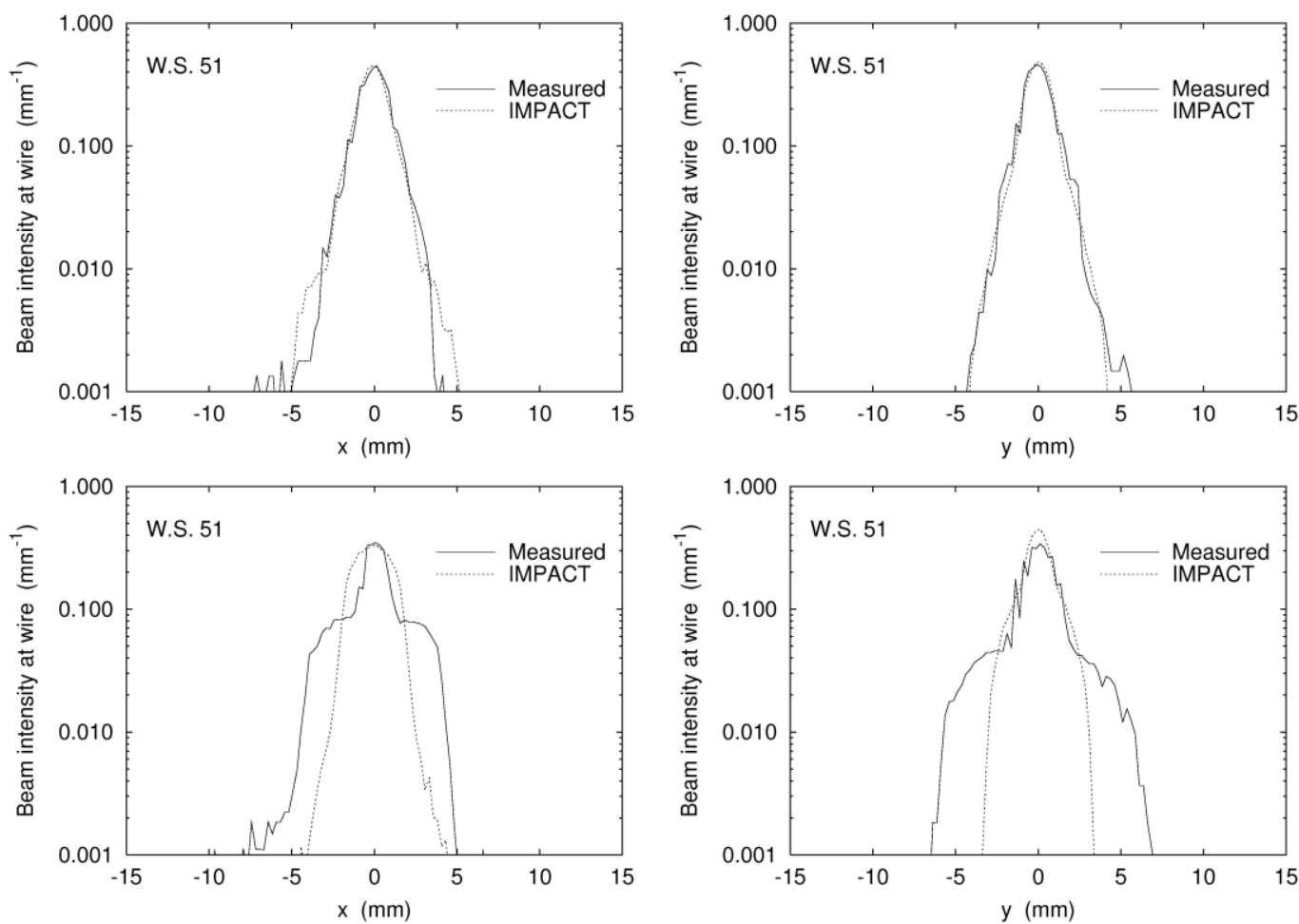

FIG. 2. Comparison of measured and simulated profiles at the final wire scanner. Top row shows the matched case and the bottom row shows the mismatched case for mismatch parameter $\mu=1.5$ as defined in Ref. 8. Both cases used an input beam determined by fitting the matched data to profiles at wire scanners 4 through 26.

data simultaneously, and comparing the initial distributions in detail. The fitting code used an older version of IMPACT. We plan to repeat the fitting using the latest version of IMPACT, which will enable us to study different boundary conditions in the space charge computations. This may lead to a better characterization of the RFQ exit beam, and an improved overall analysis of the halo experiment.

\section{ACKNOWLEDGMENTS}

We acknowledge the help of Dr. L. Young for providing an initial distribution from the LEBT/RFQ simulation. We acknowledge the work on the experiment by the rest of the beam-halo scientific team, C. K. Allen, K. C. D. Chan, P. L. Colestock, K. R. Crandall, J. D. Gilpatrick, J. D. Schneider, M. E. Schulze, R. L. Sheffield, and H. V. Smith. Part of the simulations were performed on the supercomputers at the National Energy Research Scientific Computing Center and the Center for Computational Sciences at Oak Ridge National Laboratory. This work was supported by the U.S. Department of Energy.

\section{REFERENCES}

[1] J. Qiang, R.D.Ryne, S. Habib, and V. Decyk, J. Comput. Phys. 163, 434 (2000).
[2] H. Vernon Smith, J. D. Schneider, and R. Sheffield, Proc. 2001 Part. Accel. Conf., IEEE Catalog No. 01CH37268, 3296-3298.

[3] J.Qiang, et al., Phys. Rev. ST Accel. Beams, 5, (124201) (2002).

[4] T.P.Wangler et al., Proc. 2001 Part. Accel. Conf., IEEE Catalog No. 01CH37268, 2923-2925;

P.L.Colestock et al., Proc. 2001 Part. Accel. Conf., IEEE Catalog. No. 01CH37268, 170-172; M. Schulze et al, Proc. 2001 Part. Accel. Conf., IEEE Catalog No. 01CH37268, 591-593.

[5] J.D.Gilpatrick et al., Proc. 2001 Part. Accel. Conf., IEEE Catalog No. 01CH37268, 525-527.

[6] T.P.Wangler, K.R.Crandall, R. Ryne, and T.S.Wang, Phys.Rev. ST Accel. Beams 1, (084201)1998.

[7] M. Reiser, Theory and Design of Charged Particle Beams, (Wiley, New York, 1994), pp. 470-473,; M. Reiser, J.Appl. Phys. 70, 1919 (1991).

[8] C.K.Allen, et al., Phys.Rev.Lett. 89, No.21, (214802) 2002.

[9] W.P.Lysenko et al., presented at the Int. Comp. Accel. Phys. Conf. 2002, Michigan State University, East Lansing MI, Oct. 15-18, 2002. 